\title{
Epidemiological and clinical risk factors for non- erosive reflux disease, erosive esophagitis and Barrett's esophagus in Russia
}

\begin{abstract}
The aim of the study was to identify the epidemiological and clinical factors predisposing to non-erosive reflux disease (NERD), erosive esophagitis (EE) and Barrett's esophagus.

Materials and methods: 1433 patients with GERD from Moscow, Moscow region, St Petersburg and Leningrad region were studied. During the study complaints and anamnestic data were gathered, anthropometry, tests for $H$. pylori infection and upper endoscopy were performed. Spearman rank correlation was used to identify the correlation of clinical characteristics with NERD, EE and Barrett's esophagus. 880 patients with GERD (407 with NERD, 443 with EE, 27 with Barrett's esophagus, 3 with esophageal stricture) were selected for correlation analysis due to the presence of all necessary parameters.
\end{abstract}

Results: The prevalence of two main GERD phenotypes - EE and NERD - was approximately equal in the patients of Russian megapolises. Male gender was significantly negatively correlated with NERD development $(\mathrm{p}=0.002011)$. An inverse correlation was found between NERD and older age groups $(\mathrm{p}=0.002459)$, high weight circumference (WC) $(\mathrm{p}=0.003733)$, long duration of GERD symptoms $(\mathrm{p}=0.012984)$, the results of the GerdQ $\geq 8$ points $(\mathrm{p}=0.040030)$. In the group of patients with $\mathrm{EE}$ grade $\mathrm{A}-\mathrm{B}$, a significant inverse correlation was revealed with age over 40 years $(\mathrm{p}=0.002708)$, high body mass index (BMI) $(\mathrm{p}=0.020957)$, and high WC $(\mathrm{p}=0.001293)$. The male gender $(\mathrm{p}=0.000108)$ and the duration of GERD symptoms more than 3 years $(\mathrm{p}=0.028292)$ were significantly associated with severe grades of EE development. The male gender $(\mathrm{p}=0.007505)$ and not using of PPIs over the past 6 months ( $\mathrm{p}=0.015930$ ) were positively associated with Barrett's esophagus.

Conclusion: Female gender, young age, normal BMI and WC, short duration of symptoms and GERD-Q less than 7 points were positively associated with NERD compared with EE among Russian patients. Male patients with long-term GERD symptoms who do not take PPIs are at high risk of Barrett's esophagus.

Keywords: gastroesophageal reflux disease, erosive esophagitis, non-erosive reflux disease, proton pump inhibitors, heartburn, Barrett's esophagus
Volume 12 Issue 6 - 202I

Igor Maev, ' Igor Bakulin, ${ }^{2}$ Dmitriy Bordin, ${ }^{1,3,4}$ Nataliya Bakulina, ${ }^{2}$ Inna Oganezova, ${ }^{2}$ Ekaterina Skazyvaeva, ${ }^{2}$ Maria Skalinskaya, ${ }^{2}$ Yulia Embutnieks, ${ }^{4}$ Roman Plavnik, ${ }^{5}$ Lyudmila Fedulenkova, ${ }^{4}$ Sergey Tikhonov, ${ }^{2}$ Mariia Zhuravleva, ${ }^{2}$ Roman Vasiliev ${ }^{6}$

'Moscow State University of Medicine and Dentistry n.a.A.I. Yevdokimov, Russia

${ }^{2}$ North-Western State Medical University n.a. I.I. Mechnikov, Russia

${ }^{3}$ Tver State Medical University, Russia

${ }^{4} \mathrm{SBHI}$ «Moscow clinical scientific and practical center n.a.A.S.

Loginov» of the Moscow Healthcare Department, Russia

Isocarb LLC, Russia

'Multidisciplinary Clinics «OSNOVA», Saint-Petersburg, Russia

Correspondence: Roman Vasiliev, Multidisciplinary Clinics "OSNOVA», Saint-Petersburg, Russia, Email doctor.vrv@yandex.ru

Received: October 28, 2020 | Published: November 19, 202
Abbreviations: GERD, gastroesophageal reflux disease; EE, erosive esophagitis; NERD, non-erosive reflux disease; PPIs, proton pump inhibitors

\section{Key summary}

a. GERD is a widespread disease, which is associated with increased risk of Barrett's esophagus and esophageal adenocarcinoma.

b. However, no data on risk factors predisposing to the development of non-erosive reflux disease, erosive esophagitis and Barrett's esophagus in Russia is available.

I. What are the significant and/or new findings of this study?

a. We showed that female gender, young age, normal BMI and WC, short duration of GERD symptoms and Gerd-Q results $<7$ points were positively associated with NERD.

b. We showed that male patients with long-term GERD symptoms who do not use PPIs are at high risk of Barrett's esophagus.

\section{Introduction}

Gastroesophageal reflux disease (GERD) is one of the most common diseases in developed countries. Many studies have shown that GERD has a high worldwide prevalence varying from $8 \%$ to $33 \%$ and involves all age groups and both genders. ${ }^{1}$ Moreover, the frequency of GERD is constantly increasing, which may be associated with an increase in the incidence of obesity and other metabolic diseases. Nowadays the prevalence of GERD in North America and in Western Europe is up to $27.8 \%$ and $25.9 \%$ respectively. ${ }^{2}$ In Russia, GERD prevalence ranges from 13.3 to $23.6 \%$ in different regions. ${ }^{3}$

According to the modern concept of GERD spectrum, patients with GERD can be divided into three main phenotypes: non-erosive reflux disease (NERD), erosive esophagitis (EE), and GERD with complications, such as Barrett's esophagus, esophageal stricture, esophageal adenocarcinoma and pulmonary fibrosis., ${ }^{2,45}$ The NERD phenotype represents the majority of patients with GERD, and is diagnosed in approximately $70 \%$ of the patients, while the EE prevalence is about $30 \% .{ }^{6}$ Moreover, based on the upper endoscopy and impedance $\mathrm{pH}$-monitoring results patients with NERD can be subdivided into disorders with different pathways and therapeutic options: NERD with catarrhal esophagitis, endoscopically negative GERD with pathological acid exposure time (AET) and positive symptom-association probability (SAP) and hypersensitive esophagus with normal AET and positive SAP. The functional heartburn, which is characterized by negative SAP, normal AET and absence of esophagus mucosa lesions, is included into functional disorders, so- 
called Gut-Brain axis disorders. ${ }^{6-8}$ GERD phenotypes can succeed each other, thus in $10 \%$ of cases NERD can transform into EE. ${ }^{2}$

There is no «gold standard» for the diagnosis of GERD nowadays. Most of the clinical practice guidelines have developed stepwise diagnostic algorithms depending on the initial characteristics of the patient. $^{9-12}$ The main diagnostic methods for GERD in real clinical practice include The Reflux Disease Questionnaire (GerdQ), PPI test, empirical proton pomp inhibitor (PPI) therapy for 12weeks, upper endoscopy and biopsies, impedance $\mathrm{pH}$-monitoring, but each of these methods has its own limitations. Some studies have showed that Helicobacter pylori (H. pylori) infection is associated with the development of GERD. According to these data it is recommended to identify $H$. pylori among patients with GERD and initiate eradication therapy if the result is positive. H. pylori infection is widespread in Russia. According to our data around $50 \%$ of patients with different stomach diseases and $42 \%$ of patients with dyspepsia are infected with $H$. pylori infection in Russia. ${ }^{13,14}$ And the prevalence of $H$. pylori infection among doctors (general practitioners) in Saint-Petersburg is around $60 \% .{ }^{15}$ But some researches deny any relationship between GERD and H. pylori. ${ }^{16,17}$

Knowledge of risk factors for NERD, EE and Barrett's esophagus development helps to determine optimal treatment and surveillance for each patient with heterogeneous GERD phenotypes. Risk factors associated with the development of GERD were studied in different researches. ${ }^{5,9-11}$ Mazzoleni F. et al. in randomized clinical trial HEROES-GERD showed that combination of increased body mass index and waist circumference predicts new-onset esophagitis. ${ }^{17}$ The 12-month follow-up esophagitis rates for overweight and normal body weight patients were $13.6 \%(29 / 213)$ and $6.0 \%(10 / 167)$, respectively $(\mathrm{p}=0.015)$; rates for patients with and without increased baseline waist circumference were $15.4 \%(24 / 156)$ and $6.7 \%$ $(15 / 224)$, respectively $(p=0.006)$. Dietary habits (fat and spice food, carbonated drinks, fermentable carbohydrates), hiatal hernia, concomitant gastroenterological diseases, concomitant medications (antibiotics, calcium-channel blockers, bisphosphonates), genetic factors ${ }^{18}$ and psychological status of the patient ${ }^{19}$ were determined to be associated with GERD occurrence. According to Schmidt M. et al. demographic, lifestyle, and clinical factors as well as GERD symptoms were associated with Barrett's esophagus development, suggesting that a combination of risk factors could be useful in developing individualized screening efforts for patients with Barrett's esophagus and GERD in Germany. ${ }^{20}$

The first-line medical therapy of GERD is anti-secretory drugs, most commonly PPIs. ${ }^{17}$ However, clinical practice data showed that about half of patients were refractory to PPI. In $20-50 \%$ of cases symptoms of GERD and/or endoscopic lesions of esophagus mucosa persisted during treatment with PPI for 8 weeks. ${ }^{17,21}$ In $50-90 \%$ of patients, during the PPI therapy or within a year after the withdrawal of PPIs, the symptoms of the disease recurred. ${ }^{22,23}$ Furthermore, there are differences between responses to PPI therapy in patients with EE and typical reflux symptoms and patients with NERD: the response to standard dose of PPI in EE patients is about 55.5\%, whereas the NERD patients have a rate of $36.7 \% .^{2,24}$ There are several explanations for PPI non-response or partial response, such as low compliance to prescribed therapy and inappropriate timing of administration, which may reduce the effect of the drug; individual pharmacokinetics of PPIs; weakly acidic reflux and remaining of alkaline reflux during PPI therapy. ${ }^{22-25}$ Non-GERD conditions may also cause symptoms similar to GERD: Crohn's disease of upper GI tract, eosinophilic esophagitis, esophageal achalasia and others. In that cases non-response to PPI treatment may also occur.
The aim of the study was to identify the epidemiological and clinical factors predisposing to non-erosive reflux disease (NERD), erosive esophagitis (EE) and Barrett's esophagus in Russian population of patients.

\section{Materials and methods}

The study included 1433 patients with GERD from Moscow, Moscow region and St. Petersburg. Written, informed consent was obtained from each patient included in the study. This study conformed to the ethical guidelines of the 1975 Declaration of Helsinki and was approved by the local ethical committee of Federal State Budgetary Educational Institution of Higher Education "North-Western State Medical University named after I.I. Mechnikov" of the Ministry of Health of the Russian Federation on 04.04.2018.

There were 595 (42\%) men and 838 (58\%) women with the average age $48 \pm 16$ years (from 7 to 91 years). The study design included 3 visits with an interval of 4 weeks, during which complaints and anamnestic data were gathered, anthropometry, upper endoscopy, tests for $H$. pylori infection and adherence evaluation were performed. For anti-secretory effect rabeprazole was prescribed for most of the patients $(\mathrm{n}=1333,96 \%)$.

The obtained data were analyzed by parametric and non-parametric statistical methods with statistical programme «SPSS». Correlation analysis with Spearman rank correlation was used to identify the correlation of clinical characteristics with NERD, EE and Barrett's esophagus. 880 patients with GERD (407 with NERD, 443 with EE, 27 with Barrett's esophagus, 3 with esophageal stricture) were selected for correlation analysis due to the presence of all necessary parameters.

As potential risk factors for NERD, EE and Barrett's esophagus development were suggested the following: age, gender, body mass index (BMI) and waist circumference (WC), smoking, H. pylori presence, hiatal hernia, duration of symptoms, previous PPIs therapy, concomitant therapy.

\section{Results}

Analysis of age and gender characteristics of patients showed that GERD was diagnosed in all age groups. Among male patients the most common was the age group 30-39 years (30\%), among female - age group 60-69 years $(23 \%)$. These results correspond with published data, according to which the incidence of GERD is higher in the older age groups.

The number of patients with the high BMI was 895 (62.5\%). A direct moderate association between BMI and the age of patients was found (Spearman's ratio $=0.39$ ). The high value of $\mathrm{WC}$ was detected in $193(39 \%)$ men and in $380(39 \%)$ women.

Overweight and obesity are known to be associated with the development of GERD. Mazzoleni F. et al. ${ }^{17}$ in a randomized clinical trial of patients with functional dyspepsia HEROES-GERD $(n=404)$ showed that EE is significantly more frequent in patients with overweight $(\mathrm{p}=0.015)$ compared with patients with normal body weight $(13.6 \%$ and $6.0 \%$ respectively). Significant differences were also found in the prevalence of EE depending on the WC: $15.4 \%$ in patients with high $\mathrm{WC}$ values and $6.7 \%$ in patients with normal $\mathrm{WC}$ values $(\mathrm{p}=0.006){ }^{17}$

914 (65\%) patients didn't smoke, 200 (14\%) patients were smokers in the past, $299(21 \%)$ patients were current smokers. According to $\chi 2$ test no significant differences were found between smoking and the 
grade of EE (A and B-D). According to published data the role of smoking in the development of GERD is not well defined, while the carcinogenic effect of tobacco smoking is well established.

$130(9 \%)$ patients were taking concomitant therapy, which included low doses of aspirin, NSAIDs, calcium-channel blockers, beta-blockers, statins, and a combination of these drugs. In the presence of concomitant therapy all patients were diagnosed with erosive esophagitis, most of whom (534 patients; 93\%) revealed stages $\mathrm{A}$ and $\mathrm{B}$, which required differential diagnosis with druginduced esophageal injury.

According to the Gerd-Q results moderate and high probability of GERD was determined in $635(44 \%)$ and $560(39 \%)$ patients respectively. Gerd-Q test results were significantly higher in male patients compared with female patient; in the group of patients older than 45years compared with patients younger than 45years, as well as in the group of patients with high BMI $(>25)$ compared with the patients with normal BMI range (Mann-Whitney U-test, $\mathrm{p}<0.05$ ).

According to Gerd-Q test, a low probability of GERD was determined in $238(17 \%)$ patients. This data reflected the possibility of esophageal lesions without any reflux symptoms. In patients with EE significant difference between Gerd-Q results and EE grade was revealed: in patients with EE grade B-D results of Gerd-Q were significantly higher compared to patients with EE grade A (the MannWhitney test, $\mathrm{p}<0.05$ ). The sensitivity of Gerd-Q test in the diagnosis of EE was $82 \%$, specificity - $16 \%$. Thus, high probability of GERD according to the Gerd-Q results reflected the risk of high-grade esophageal lesions, but was not applicable for differential diagnosis between EE, NERD and other disorders of the gastrointestinal (GI) tract.

Table I Spearman Rank Order Correlations for NERD

\begin{tabular}{lllll}
\hline Variables & Valid & Spearman & t(N-2) & p-level \\
\hline no visible changes of esophageal mucosa \& male gender & 880 & $-0,103983$ & $-3,09793$ & 0,002011 \\
Catarrhal esophagitis \& male gender & 880 & $-0,043592$ & $-1,29290$ & 0,196385 \\
Catarrhal esophagitis \& smoking & 880 & 0,000864 & 0,02560 & 0,979583 \\
Catarrhal esophagitis \& age group & 880 & $-0,101963$ & $-3,03709$ & 0,002459 \\
Catarrhal esophagitis \& BMI & 880 & $-0,065762$ & $-1,95283$ & 0,051158 \\
Catarrhal esophagitis \& WC & 880 & $-0,097660$ & $-2,90768$ & 0,003733 \\
Catarrhal esophagitis \& Duration of symptoms & 880 & $-0,083715$ & $-2,48929$ & 0,012984 \\
Catarrhal esophagitis \& GerdQ & 880 & $-0,069237$ & $-2,05649$ & 0,040030 \\
Catarrhal esophagitis \& previous PPIs treatment & 880 & 0,023804 & 0,70553 & 0,480666
\end{tabular}

Table 2 Spearman Rank Order Correlations for EE

\begin{tabular}{lllll}
\hline Variables & Valid & Spearman & $\mathbf{t ( N - 2 )}$ & p-level \\
\hline EE grade A-B \& Male gender & 880 & $-0,030047$ & $-0,89074$ & 0,373315 \\
EE grade A-B \& Smoking & 880 & $-0,02003 \mathrm{I}$ & $-0,59364$ & 0,552903 \\
EE grade A-B \& Age group & 880 & $-0,100983$ & $-3,0076 \mathrm{I}$ & 0,002708 \\
EE grade A-B \& BMI & 880 & $-0,07782 \mathrm{I}$ & $-2,31293$ & 0,020957 \\
EE grade A-B \&WC & 880 & $-0,108300$ & $-3,22802$ & 0,001293 \\
EE grade A-B \& Duration of symptoms & 880 & $-0,035362$ & $-I, 04847$ & $0,29471 \mathrm{I}$ \\
EE grade A-B \& GerdQ & 880 & $-0,036374$ & $-I, 07850$ & 0,281106
\end{tabular}

Citation: Maev I, Bakulin I, Bordin D, et al. Epidemiological and clinical risk factors for non-erosive reflux disease, erosive esophagitis and Barrett's esophagus in Russia. Gastroenterol Hepatol Open Access. 202I;I2(6):I76-I80. DOI: 10.15406/ghoa.2021.12.00482 


\begin{tabular}{lllll} 
Table Continued... & Valid & Spearman & $\mathbf{t}(\mathbf{N}-2)$ & p-level \\
\hline Variables & 880 & $-0,0009$ II & $-0,02699$ & 0,978477 \\
\hline EE grade A-B \& previous PPIs treatment & 880 & 0,130162 & 3,889924 & 0,000108 \\
EE grade C-D \& Male gender & 880 & 0,036444 & 1,080596 & 0,280174 \\
EE grade C-D \& Smoking & 880 & 0,015645 & 0,463639 & 0,643021 \\
EE grade C-D \& Age group & 880 & 0,019657 & 0,582572 & 0,560331 \\
EE grade C-D \& BMI & 880 & 0,000452 & 0,013399 & 0,989312 \\
EE grade C-D \& WC & 880 & 0,073937 & 2,196841 & 0,028292 \\
EE grade C-D \& Duration of symptoms & 880 & 0,051355 & 1,523709 & 0,127941 \\
EE grade C-D \& GerdQ & 880 & $-0,015325$ & $-0,454151$ & 0,649832 \\
EE grade C-D \& previous PPls treatment & &
\end{tabular}

The male gender $(p=0.007505)$ and not-using of PPIs over the last 6 months $(p=0.015930)$ were positively correlated with Barrett's esophagus development

In the group of patients with $\mathrm{EE}$ grade $\mathrm{A}-\mathrm{B}$, a significant inverse correlation was revealed with age over 40years $(\mathrm{p}=0.002708)$, high body mass index, BMI, $(\mathrm{p}=0.020957)$, and high WC $(\mathrm{p}=0.001293)$. In patients with the GERD duration of more than 5 years, the risk of having a high grade erosive lesions (B, C or D) was significantly higher than in patients with the disease duration of less than 5years $(\mathrm{OR}=1.85)$. The male sex $(\mathrm{p}=0.000108)$ and the duration of symptoms more than 3 years $(p=0.028292)$ were significantly related to severe grades of EE (Table 1). The male gender $(\mathrm{p}=0.007505)$ and not-using of PPIs over the last 6 months $(\mathrm{p}=0.015930)$ were positively correlated with Barrett's esophagus development.

\section{Discussion}

Among the examined population of patients with GERD, two main phenotypes of the disease - EE and NERD - were equally found, although, according to published data, in the last decade the prevalence of NERD is higher than EE. ${ }^{1-2}$.

The obtained results indicated a high probability of developing NERD in women, young age patients, with WC within the normal range, duration of symptoms less than 3 years, and low GerdQ scores $<7$ points. These parameters can be used by gastroenterologist while choosing the optimal diagnostic methods and therapy. In young female patients with normal WC and BMI, short duration of symptoms and low GerdQ scores, empirical course of PPIs might be considered. In case of PPI refractory GERD 24-hours pH-monitoring might be helpful.

Male older patients with high BMI and WC, especially with long duration of symptoms, are at high risk for EE development and thus need to undergo upper endoscopy at initial diagnosis without empirical PPIs therapy. For these patients dietary and lifestyle recommendations should be included in the management plan.

Male patients with long-term GERD symptoms who do not take PPIs for the last 6months are at high risk for Barrett's esophagus development, which means that they require regular endoscopic monitoring and decision on possible surgical treatment in case of the absence of the clinical and/or endoscopic effect of acid-suppression therapy.

The obtained data showed that H. pylori can contribute to more severe erosive lesions of esophageal mucosa. According to the WGO guidelines in populations with high $H$. pylori prevalence all patients with GERD should be tested for $H$. pylori and in case of positive results - eradication therapy should be prescribed.

\section{Conclusion}

The prevalence of two main GERD phenotypes - EE and NERD - is approximately equal in the population of Russian megalopolises patients. Female gender, young age, normal BMI and waist circumference, short duration of symptoms and Gerd-Q results less than 7 points were positively associated with NERD development compared with erosive esophagitis among Russian patients. Male patients with long-term GERD symptoms who do not take PPIs are at high risk of Barrett's esophagus development.

\section{Acknowledgments}

We would like to express your gratitude to all gastroenterologists, who were involved in patients' data collection.

\section{Conflicts of interest}

The authors declare that there are no conflicts of interest.

\section{Funding}

None.

\section{References}

1. Gyawali CP, Kahrilas PJ, Savarino E, et al. Modern diagnosis of GERD: the Lyon Consensus. Gut. 2018;67(7):1351-1362.

2. Ribolsi M, Giordano A, Guarino MPL, et al. New classifications of gastroesophageal reflux disease: an improvement for patient management? Expert Rev Gastroenterol Hepatol. 2019;13(8):761-769.

3. Lazebnik LB, Masharova AA, Bordin DS, et al. Results of a multicenter trial "Epidemiology of Gastroesophageal Reflux Disease in Russia" (MEGRE). Ter Arkh. 2011;83(1):45-50.

4. Bandyopadhyay N, Fass R, Yamasaki T, et al. Pocket Handbook of Esophageal Disorders. Chapter 11. Gastroesophageal Reflux Disease (GERD). Switzerland: Springer Nature; 2019.p. 85

5. Bakulin IG, Bordin DS, Drapkina OM, et al. Phenotypes od Gastroesophageal Reflux Disease in real clinical practice. Consilium Medicum. 2019;21(8):15-22.

6. Vakil N, van Zanten SV, Kahrilas P, et al. The Montreal definition and classification of gastroesophageal reflux disease: a global evidence-based consensus. Am J Gastroenterol. 2006;101:1900-1920.

7. Miwa H, Kondo T, Oshima T, et al. Esophageal sensation and esophageal hypersensitivity - overview from bench to bedside. J Neurogastroenterol Motil. 2010;16(4):353-362. 
8. Dickman R, Maradey-Romero C, Fass R. The role of pain modulators in esophageal disorders - no pain no gain. Neurogastroenterol Motil. 2014;26(5):603-610.

9. Hiltz SW, Black E, Modlin IM, et al. AGA Medical Position Statement on the Management of Gastroesophageal Reflux Disease. Gastroenterology. 2008;135(4):1383-1391, 1391.e1-5.

10. Katz PO, Gerson LB, Vela MF. ACG practice guidelines for the diagnosis and management of gastroesophageal reflux disease. Am J Gastroenterol. 2013;108:308-328.

11. Fock KM, Talley N, Goh KL, et al. Asia-Pacific consensus on the management of gastro-oesophageal reflux disease: an update focusing on refractory reflux disease and Barrett's oesophagus. Gut. 2016;65(9):14021415 .

12. Roman S, Gyawali CP, Savarino E, et al. Ambulatory reflux monitoring for diagnosis of gastro-esophageal reflux disease: update of the Porto consensus and recommendations from an international consensus group. Neurogastroenterol Motil. 2017;29:1-15.

13. Zakharova NV, Simanenkov VI, Bakulin I, et al. Prevalence of Helicobacter pylori infection in gastroenterological patients in SaintPetersburg. Pharmateca. 2016;33-39.

14. Bakulina N, Bakulin I, Ilchishina T. Epidemiological study on Helicobacter pylori infection among patients with dyspepsia in Saint-Petersburg (Russia). Helicobacter. 2018;23(Suppl. 1):e12525.

15. Bakulina N, Simanenkov VI, Bakulin I, et al. Prevalence of H. pylori infection among physicians. Experimental and Clinical Gastroenterology. 2017;(12):20-24.

16. Mari A, Mahroum N, Bragazzi NL, et al. Demographics and Clinical and Endoscopic Characteristics of Patients with Helicobacter pylori Infection and Gastroesophageal Reflux Disease: A Case-Control Study. Gastroenterol Res Pract. 2019;2019:3819893.
17. Mazzoleni F, Mazzoleni LE, de Magalhães Francesconi CF, et al. Potential roles of Helicobacter pylori treatment, body mass index and waist circumference in the causation of erosive esophagitis: a randomized clinical trial (HEROES-GERD). Int J Obes (Lond). 2020;44(1):147-158.

18. An J, Gharahkhani P, Law MH, Gastroesophageal reflux GWAS identifies risk loci that also associate with subsequent severe esophageal diseases. Nat Commun. 2019;10(1):4219.

19. Jang SH, Ryu HS, Choi SC, et al. Psychological factors influence the gastroesophageal reflux disease (GERD) and their effect on quality of life among firefighters in South Korea. Int J Occup Environ Health. 2016;22(4):315-320.

20. Schmidt M, Ankerst DP, Chen Y, et al. Epidemiological risk factors in a comparison of a Barrett Esophagus Registry (BarretNET) and a case control population in Germany. Cancer Prev Res (Phila). 2020;13(4):377384

21. Blackshaw LA, Bordin DS, Brock C, et al. Pharmacologic treatments for esophageal disorders. Ann N Y Acad Sci. 2014;1325:23-39.

22. Boeckxstaens GE, Smout A. Systemic review: role of acid, weakly acid and weakly alkaline reflux in gastro-oesophageal reflux disease. Alim Pharmacol Ther. 2010;32 (3):334-343.

23. Cicala M, Emerenziani S, Guarino MP, et al. Proton pump inhibitor resistance, the real challenge in gastro-esophageal reflux disease. World J Gastroenterol. 2013;19(39):6529-6535.

24. Furuta T, Sugimoto M, Kodaira C, et al. CYP2C19 genotype is associated with symptomatic recurrence of GERD during maintenance therapy with low-dose lansoprazole. Eur J Clin Pharmacol. 2009;65(7):693-698.

25. Richter JE. How to manage refractory GERD. Nat Clin Pract Gastroenterol Hepatol. 2007;4(12):658-664. 